\title{
First-principles Study of Electronic and Magnetic Properties of Manganese Decorated Graphene
}

\author{
Bishnu Prasad Paudel, Nurapati Pantha, and Narayan Prasad Adhikari* \\ Central Department of Physics, Tribhuvan University \\ Kirtipur, Kathmandu, Nepal \\ *Correspondence to: npadhikari@gmail.com
}

\begin{abstract}
The functionalization of graphene by the addition of Manganese (Mn) atom to its surface has been investigated computationally by using density-functional theory (DFT) based first-principles method within DFT-D2level of approximations. The calculations have been computed employing the Quantum ESPRESSO codes. The stability, geometrical structures, electronic and magnetic properties of pure and Mn adatom graphene systems have been studied. From the information of adsorption energies of Mn atom on the different sites of graphene, the top site is found to be the most favorable one for its adsorption. Present study finds that the London dispersion interaction plays a major role in the weak binding of Mn on graphene. The study of electronic and magnetic properties of Mn decorated graphene shows that the conduction and valence band are overlapped with finite density of states (DOS) at Fermi level. The dissimilar DOS for up and down spin calculations quantify magnetic moment as $5.48 \mu_{B}$ which is consistent with the previous study
\end{abstract} Keywords: DFT; Magnese; quantum espresso; band structure

\section{Introduction}

Graphene, the well-publicized twodimensional carbon allotrope, is a versatile material with many Superlatives to its name. In the material view, graphene has been considered as a kind of ambidextrous and unique building block for functional materials [e.g., Compton and Nguyen, 2010]. It has attracted strong scientific and technological interest because of its unique physical/chemical properties (high surface area [e. g. Novoselov et al., 2005], excellent conductivity and mechanical strength [e.g., Geim, 2009; Murali et al., 2009] and potential of low production cost [e.g., Novoselov et al., 2005; Wolf, 2013]. Graphene has shown great potential in many fields, such as electronics and energy storage. Theoretical and experimental studies

have shown that functionalized graphene can tailor the physical/chemical properties of graphene and open the possibilities of new physics on graphene [e.g., Geim, 2009]. It has been considered a desirable material for the post-silicon age [e.g., Novoselov et al., 2005; Novoselov et al., 2004; Novoselov et al., 2007]. The most entertained properties of the graphene are an ambipolar electric field effect along with ballistic conduction of carriers [e.g., Novoselov et al., 2004], tunable band gap [e.g., Zhang et al., 2009], and high elasticity [e.g., Lee et al., 2008]. Evincing all these enormous potential in the electronic device community researchers led their focus on its use on various practical purpose, for example, field-effect transistor, transparent electrode, super small transistors, 
super dense data storage, increased energy storage and solar cell efficiency etc. [e.g., Wolf et al., 2013].

The interaction of graphene with foreign particles can be understood in two types of adsorptions: chemisorption (due to local electronic rearrangement) and physisorption (due to non-local electronic interactions, named van der Waals forces) [e.g., Ferrari et al., 2010]. The nature and strength of interaction between the adatom and graphene is determined by the electronic/magnetic properties of the interacting particles [e.g., Mao et al., 2008]. Because of the strong electron-electron interactions in between graphene and transition metal atoms (TMs) with partially filled d-orbital, the materials thus formed are more susceptible to inducing a variation in the spin electronic structure. The tuning of the electronic structure in TMadsorbed graphene may open new routes for designing spintronics devices [e.g., Mao and Zhong, 2008] to investigate how its spin electronic structure changes by adsorbing TM adatoms on its surface. The TMs like Mn, which shows antiferomatism [e.g., Wu et al., 2010], is one of the interesting adatoms in nanostructures [e.g., Mao et al., 2008] to study the magnetic properties. Manganese-oxides attract considerable interest because of its use in the development of magnetic memory and switching device [e.g., Moritomo et. al., 1996].

The adsorptions of some of the alkali and transition metal atoms on graphene have been already considered (at this department and outside) to study their geometrical and electronic properties [e.g., Pantha et al., 2014; Belbase, 2013; Khaniya, 2013; Lamichhane et al., 2014; Valencia et al., 2010; Chan et al., 2008]. The works reveal changes in their geometrical and electronic structure upon the adsorption of metal atoms, intending their further applications like in energy storage. However, the detail analysis of magnetic properties and the origin of their changing magnetism upon the adsorption of metal atoms on graphene have not been uncovered. In addition to the geometrical and electronic properties of Mn-added graphene, the present work reveals the origin of changing magnetism in such weakly bound systems.

\section{Computational Details}

First-principles calculations [e.g., Thijssen, 1999] are carried out to investigate the structural stability, and electronic/ magnetic properties of Mn adatom adsorption on graphene. The calculations are performed within the framework of density-functional theory (DFT) [e.g., Hohenberg and Kohn, 1964; Kohn and Sham, 1965] including van der Waals (vdW) interactions in DFT-D2 approach, implemented with the quantum ESPRESSO code [e.g., Giannozzi, et. al., 2009]. The ions and core electrons are taken care with ultra-soft pseudopotentials where as exchange-correlation part of electron-electron interactions are treated with generalized gradient approximation (GGA) formalism as described by PerdewBurke-Ernzerhof (PBE) [e.g., Perdew et al., 1996]. In the pseudopotential description, the electronic configurations $1 \mathrm{~s}, 2 \mathrm{~s}^{2} 2 \mathrm{p}^{2}$, and $3 s^{2} 3 p^{6} 4 s^{2} 3 d^{5}$ respectively for hydrogen $(H)$, carbon (C), and $\mathrm{Mn}$ are treated explicitly as valence electrons. A plane wave basis set with the kinetic energy cutoff of $35 \mathrm{Ry}$ is used for the expansion of the ground state electronic wave function. The plane waves are chosen to have a periodicity compatible with the periodic boundary conditions of the simulating cell. 


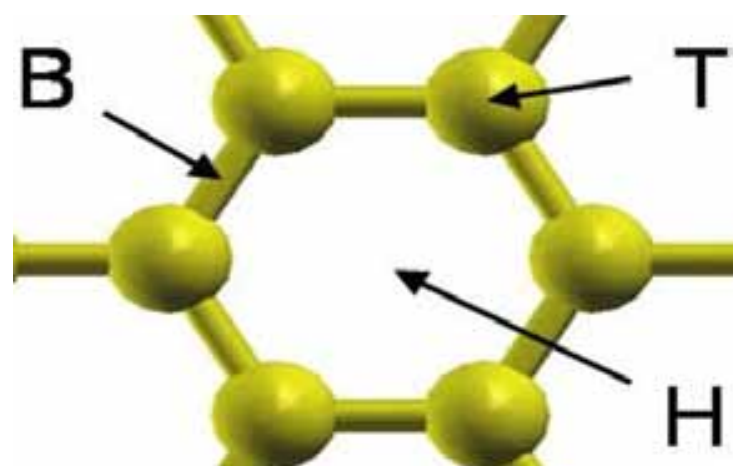

Figure 1. Schematic representation of three high symmetry adsorption sites (hollow $[\mathrm{H}]$, bridge [B] and top $[\mathrm{T}]$ ) on grapheme sheet.

Graphene contains two carbon atoms per unit cell arranged in a two-dimensional honeycomb lattice. We have used the calculated lattice constant of magnitude $2.46 \mathrm{~A}$, obtained from the convergence test, consistent to that of experimental value [e.g., Castro, 2007].

The adatom graphene system is modeled using single $\mathrm{Mn}$ adatom in the $4 \times 4$ and $5 \times 5$ super cell of graphene containing 32 and 50 number of carbon atoms. The adsorption of $\mathrm{Mn}$ on graphene is performed on three different sites of high symmetry: the top (T) site directly above the carbon atom, the hollow $(\mathrm{H})$ site at the center of hexagon, and the bridge (B) at the midpoint of $\mathrm{C}-\mathrm{C}$ bond (figure (1). For each adsorption site of the adatom-graphene, the adatom and the $\mathrm{C}$ atoms on graphene are relaxed in all $\mathrm{x}, \mathrm{y}, \mathrm{z}$ directions. To estimate the adsorption energy of $\mathrm{Mn}$ adatom, the calculations for the isolated adatom, isolated graphene and adatom graphene system are performed with the same-sized supercell of graphene. Optimization was achieved by using BFGS (Broyden-Fletcher-Goldfarb-Shanno) scheme [e.g., Fletcher,1987] until the total energy is less than $10^{-4} \mathrm{Ry}$ and force acting is less than $10^{-3} \mathrm{Ry} / \mathrm{Bohr}$. For the self-consistent total energy calculations the brillioun zone of graphene is sampled in k-space using the Monkhorst-Pack scheme [e.g., Monkhorst and Pack, 1940] with an appropriate number of mesh of k-points [e.g., Sholl and Steckel, 2009]. In order to avoid the interaction between the adatoms on adjacent super cells, vacuum length of supercell was made large enough i.e. $20 \AA$ along z- axis. The DOS of pure graphene and $\mathrm{Mn}$ adsorbed graphene is calculated for $4 \times 4$ supercell of graphene using $20 \times 20 \times 1$ mesh

\section{Results and Discussion}

In the present work, we have studied the adsorption of single $\mathrm{Mn}$ atom on different symmetry sites of $4 \times 4$, and $5 \times 5$ super cell of pure graphene. Further, the work has been extended to study the adsorption of hydrogen molecule/s on pure graphene and Mn-adsorbed graphene.

\subsection{Adsorption of Manganese on graphene}

\section{A. Adsorption energy and geometry}

The adsorption energy $(\Delta E)$ of the Mn adatom adsorption on graphene is defined as, $\Delta \mathrm{E}=\mathrm{E}_{\mathrm{Mn}}+\mathrm{E}_{\mathrm{G}}-\mathrm{E}_{\mathrm{G}+\mathrm{Mn}}$

Where, $\mathrm{E}_{\mathrm{G}+\mathrm{Mn}}, \mathrm{E}_{\mathrm{G} \text { and }} \mathrm{E}_{\mathrm{Mn}}$ are the total energy of the adatom-graphene, pure graphene and an isolated $\mathrm{Mn}$ adatom, respectively. Of the three adsorption sites $(\mathrm{H}, \mathrm{B}, \mathrm{T})$ considered, the site with the largest adsorption energy (minimum total energy) is referred to as the favored site for adsorption.

From Figure 2, it is seen that in some of the atoms like $\mathrm{Pd}$, the nearest atomic interaction gets 

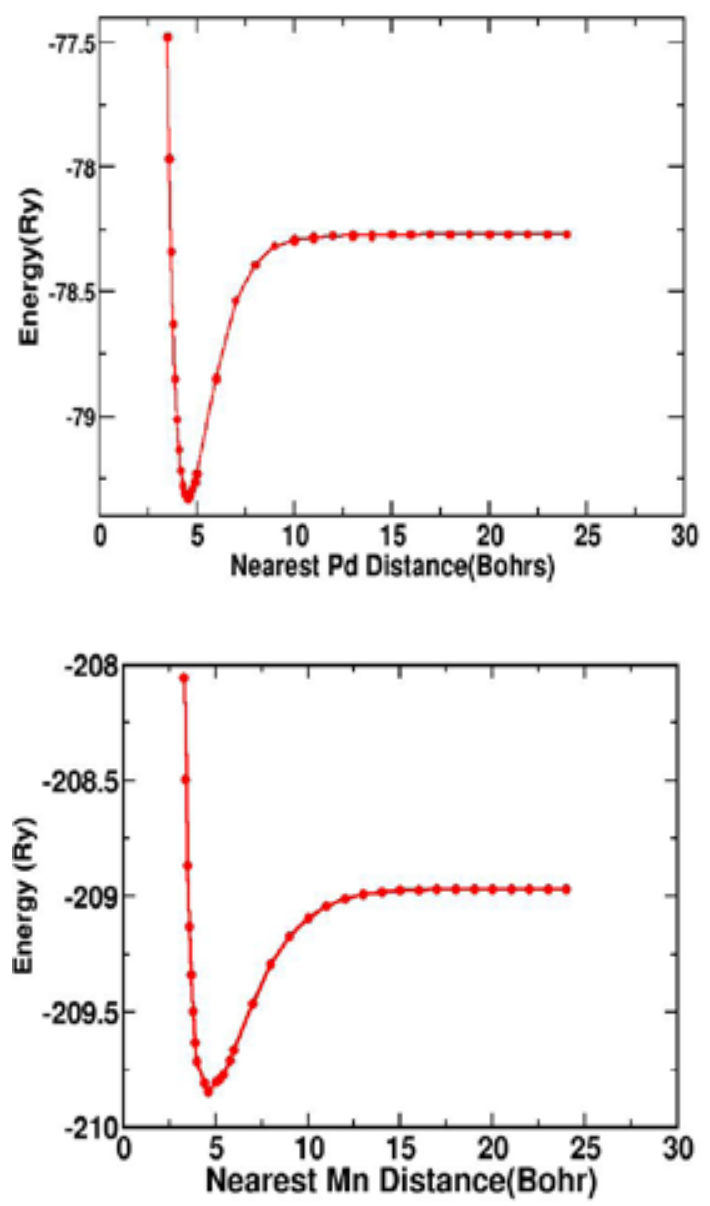

Figure 2. Variation of the total energy as a function of cell size of isolated Pd (left) and Mn (right) atoms. Two atoms ( $\mathrm{Pd}$ and $\mathrm{Mn})$ are considered for the purpose of comparison. The lattice size of cells determines the vacuum length between the nearest atoms. The figures show that $\mathrm{Pd}-\mathrm{Pd}$ interaction gets saturated approximately after 10 Bohr (which fairly agrees with the previous result [e.g., Medeiros et al., 2010], whereas that value for Mn needs to be greater than 15 Bohr.

saturated beyond 10 Bohr. However, for the atoms like $\mathrm{Mn}$, a long range atomic interaction exits and the saturation waits until 15 Bohr of Mn-Mn separations. It might be due to strong $\mathrm{d}-\mathrm{d}$ orbital interaction among $\mathrm{Mn}$ atoms [e.g.,
Medeiros et al., 2010; Fair et al., 2013]. For the purpose of comparison, the lattice parameters of $2 \times 2,3 \times 3$ and $4 \times 4$ super cells respectively are 9.30, 13.95 and 18.65 Bohr. These values imply that calculations for Pd-adsorption systems can be done in $3 \times 3$ supercell of graphene (lattice constant $13.95 \mathrm{Bohr}$ ), where as $\mathrm{Mn}$ requires at least $4 \times 4$ supercell for the reliable estimation of parameters like binding energy.

The adsorption geometry is obtained from the positions of the atoms after relaxation. The adatom height $(\mathrm{h})$ is defined as the difference in $z$ coordinate of adatom and the average of the $z$ coordinates of the carbon atoms in the graphene layer. We have also calculated the distance $\left(\mathrm{d}_{\mathrm{Ac}}\right)$ between the adatom and its nearest carbon atom. The adsorption of Mn on graphene produced significant distortion which is quantified by computing the maximum deviation in the $\mathrm{z}$ direction of the $\mathrm{C}$ atoms in the graphene layer from the average of their positions. The distortion of the graphene layer upon the adsorption is also calculated in terms of change in dihedral angles. In the present work; we have studied the adsorption of single $\mathrm{Mn}$ atom on hexagonal supercell of graphene of different size i.e. $4 \times 4,5 \times 5$ supercells containing 32 and 50 numbers of carbon atoms. The energetic and structural properties for the $\mathrm{H}, \mathrm{B}$, and $\mathrm{T}$ sites for Mn adatom adsorbed on $4 \times 4$, and $5 \times 5$ supercell of graphene are summarized in table (1)

The Table (1) clearly shows that the adsorption of $\mathrm{Mn}$ atom on different sites of pure graphene sheet is feasible. Within the same-size of graphene, its magnitude is comparable at all the tested high symmetry sites. However, it is slightly higher at the $\mathrm{T}$ site, independent to the cell-size when the size is enough to absorb isolated $\mathrm{Mn}$ without communicating to its 
mirror image. From this information, it can be concluded that $\mathrm{T}$ site is the most favorable site for the adsorption of Mn within the small energy barrier of diffusion. The adsorption energy of an adatom, in general, increases on increasing the size of the substrate [e.g., Ding et al., 2011]. The adsorption energies of $\mathrm{Mn}$ on $\mathrm{T}$ site of $4 \times 4$, and $5 \times 5$ supercell of graphene, however, are equal with magnitude $0.22 \mathrm{eV}$. This implies that the adsorption of $\mathrm{Mn}$ atom on graphene sheet with 18 or more number of carbon atoms is no more size-dependent. The magnitude of adsorption energy of Mn comparing to that for other adatoms shows that $\mathrm{Mn}$ is weakly bound on graphene, and the conclusion is consistent with the previous result [e.g., Valencia et al., 2010]. The present calculations reveal that the van der Waal's interaction plays vital role for such type of weak binding. Table (1) further shows the adatom height, the nearest carbon distance from adatom and the distortion in the graphene plane upon the adsorption of $\mathrm{Mn}$ atom on different sites of graphene. In $4 \times 4$ unit cell, the height of $\mathrm{Mn}$ from the graphene sheet is the smallest, and the distortion of the sheet is the largest upon the adsorption of $\mathrm{Mn}$ at $\mathrm{T}$ site. The parameters are consistent to approve that $\mathrm{Mn}$ is more interactive at $\mathrm{T}$ site (with the highest adsorption energy) than at the other sites.

The pure graphene sheet has bond length of $1.42 \AA$, bond angle of $120^{\circ}$, and dihedral angle of $0^{\circ}$ in between the carbon atoms. Upon the adsorption of $\mathrm{Mn}$ atom, the system gets maximum changes in bond length, bond angle and dihedral angle respectively as of 0.01 $\AA, 0.5^{\circ}$ and $1.7^{\circ}$ on $\mathrm{T}$ site of $4 \times 4$ supercell of graphene.

\section{B. Electronic structures}

In this work, we have computed the electronic density of states and band structures of pure graphene and Mn adatom graphene. The Kohn-Sham DOS is computed for Mn atom adsorbed on the $T$ site of the $4 \times 4$ supercell of graphene using 20x20x1 $\Gamma$-centered Brillouin zone-sampling. The energy eigen values are smeared by 'Marzari-Vanderbilt' [e.g., Marzari et al., 1999] smearing of width $0.001 \mathrm{Ry}$.

The DOS of pure graphene obtained from the spin polarized calculations find Dirac point at Fermi level (not shown in figure). The result is consistent with the well-known fact that the

Table 1. Structural and energetic properties of adsorption of $\mathrm{Mn}$ atom on $\mathrm{H}, \mathrm{B}$, and $\mathrm{T}$ sites of $4 \times 4$ and $5 \times 5$ supercell of graphene. The properties listed in the table are binding energy $(\Delta \mathrm{E})$, adatom height from graphene plane (h $\AA$ ), adatom-carbon distance (dAc $\AA$ ), and distortion in the graphene plane $\left(\mathrm{d}_{\mathrm{GC}} \AA\right)$.

\begin{tabular}{|l|c|c|c|c|c|}
\hline $\begin{array}{c}\text { Size of graphene } \\
\text { super cell }\end{array}$ & $\begin{array}{c}\text { Site of } \\
\text { adsorption }\end{array}$ & $\Delta \mathrm{E}(\mathrm{eV})$ & $\mathrm{h}(\AA)$ & $\mathrm{d}_{\mathrm{AC}}(\AA)$ & $\begin{array}{c}\mathrm{d}_{\mathrm{AC}} \\
\left(10^{-1} \AA\right)\end{array}$ \\
\hline & $\mathrm{H}$ & 0.16 & 2.64 & 3.34 & 4.82 \\
& $\mathrm{~B}$ & 0.19 & 2.57 & 3.18 & 5.31 \\
& $\mathrm{~T}$ & 0.22 & 2.05 & 2.21 & 5.33 \\
\hline $5 \times 5$ & $\mathrm{~T}$ & 0.22 & 2.25 & 2.21 & 2.08 \\
\hline
\end{tabular}


valence and conduction band meet at the Fermi level. The symmetric nature of DOS for spin up and spin down evince the non-magnetic nature of pure graphene.

Figure 3, [up] shows the DOS of Mn decorated graphene. The vertical dotted line in the figure represents the Fermi level as reference. In the figure, the Dirac point shifts below the Fermi level and the structure becomes non-symmetrical upon the adsorption of Mn. The asymmetry, nearby the Fermi level, can be seen around $-3.9 \mathrm{eV},-0.93 \mathrm{eV}$ and $0.8 \mathrm{eV}$ due to the contributions from different orbital's of the Mn atom (figure 3, down).

The results agree with the previous study [e.g., AlZahrani, 2012]. The non-symmetrical
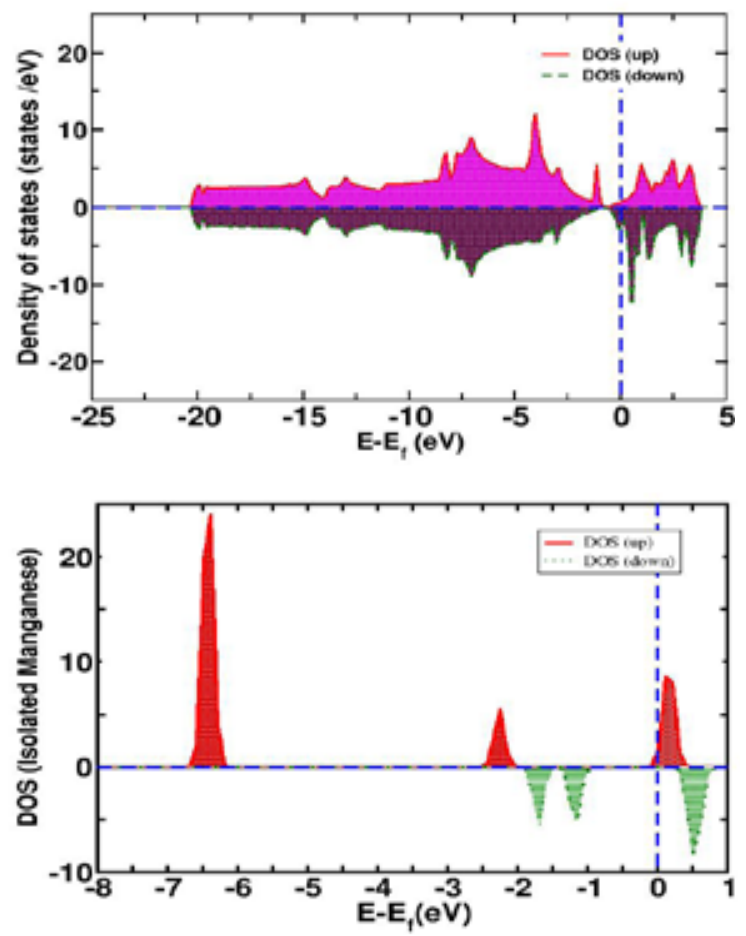

Figure 3. DOS of Mn adatom graphene (up) and that of isolated Mn atom (down). Solid line with dark shadow represents the DOS of up spin and dotted line with light shadow represents DOS of down spin.
DOS indicates the magnetic nature of $\mathrm{Mn}$ graphene system, and is quantified as $5.48 \mu_{\mathrm{B}}$ in case of Mn-added $4 \times 4$ supercell of graphene. The value comes in good agreement with the previously reported value, $5.62 \mu_{\mathrm{B}}$, within the difference of $2 \%$ [e.g., Mao et al., 2008]. The magnetization of $\mathrm{Mn}$ added graphene (5.48 $\left.\mu_{B}\right)$ is different from both of the pure graphene $\left(0 \mu_{B}\right)$ and isolated $\mathrm{Mn}$ atom $\left(5.0 \mu_{\mathrm{B}}\right)$.

To have a better understanding of the magnetism of adatom adsorpted on graphene, we illustrate the (Projected Density of States) PDOS of the adatom on Mn-graphene and DOS of isolated $\mathrm{Mn}$ atom (figure 4). In the case of $\mathrm{Mn}$ added graphene, the present calculations indicate that the system is ferromagnetic (FM) (consistent with ref. 13) with a net magnetic moment of $5.48 \mu_{\mathrm{B}}$ per unit cell. On the other hand, the net magnetic moment of isolated Mn is $5.00 \mu_{\mathrm{B}}$ per unit cell. This increment of magnetic moment of Mn-graphene system is due to rearrangement of electronic charge of $4 \mathrm{~s}$, $3 p$, and $3 d$ orbitals of the Mn (Figure 4). Having known that a defect-free graphene structure performs no magnetic moment, this value strongly suggests that the adsorption of Mn on the graphene induces the magnetic properties on the substrate.

Since the normal ground-state electronic structure of $\mathrm{Mn}$ atom has the $[\mathrm{Ar}] 3 \mathrm{~d} 54 \mathrm{~s}^{2}$ configuration, the $4 \mathrm{~s}$ shell is closed with two electrons and the exchange interaction favors as many electrons having the same spin orientation as possible ( $3 \mathrm{~d} 5 \mathrm{Mn})$. The additional low lying electrons $3 s^{2} 3 p 6$, which are considered valence electrons in the present calculations, are also filled and do not contribute to the magnetism of an isolated $\mathrm{Mn}$ atom. From the present 

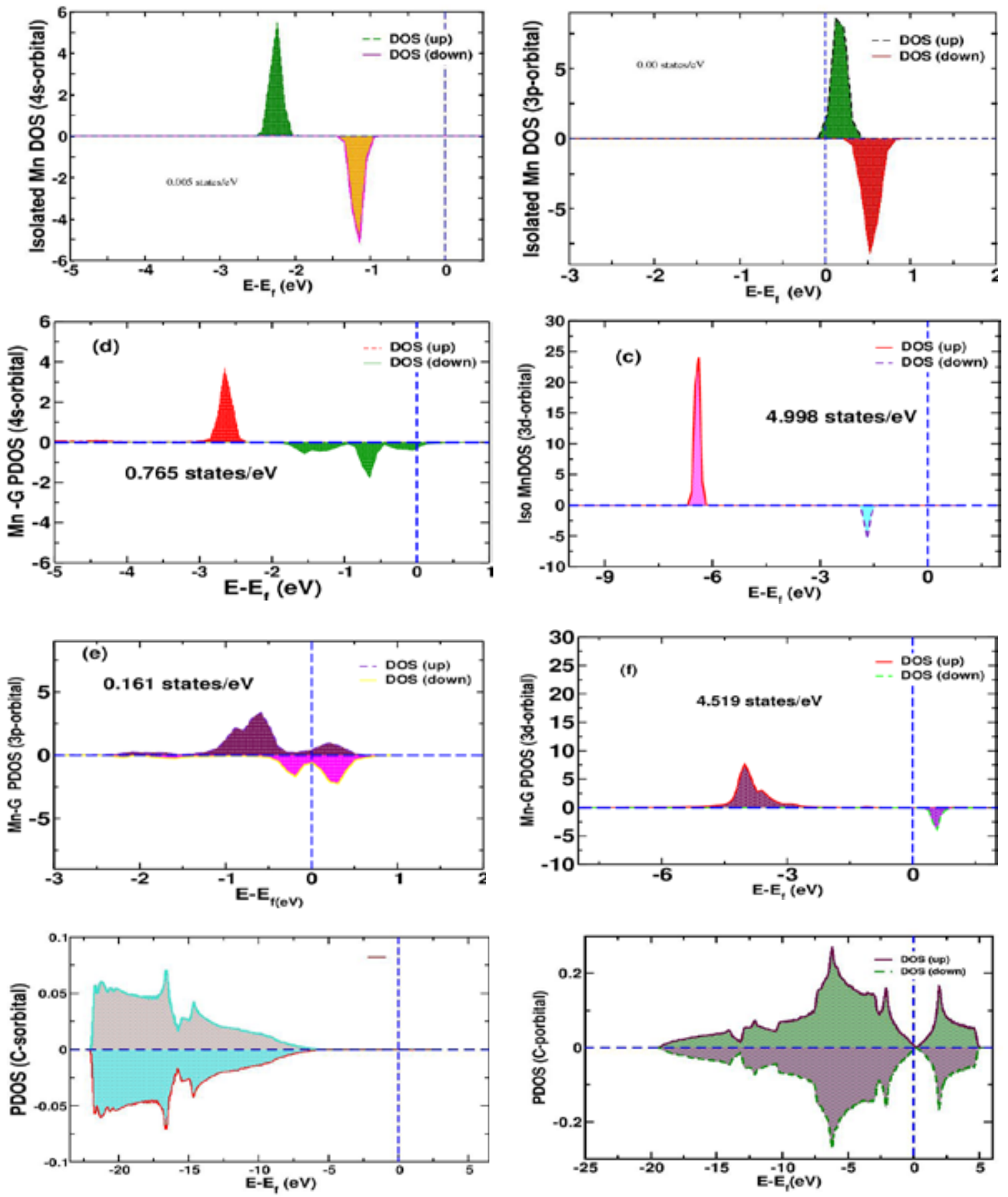

Figure 4. Partial density of states (PDOS) for spin up and spin down of s, p, and d-orbital's of isolated Mn atom (from the top), Mn atom on Mn adsorbed-graphene and $\mathrm{p}$ and s orbital's of carbon atom of graphene upon the adsorption of Mn. 
investigation, we find that the contributions of $3 \mathrm{~d}, 3 \mathrm{p}$ and $4 \mathrm{~s}$ orbitals on the net magnetization of isolated $\mathrm{Mn}$ atom can be understood from their DOS calculations where the polarized magnitudes of occupations (Lowden charge) are 4.998, 0.000 and 0.005 respectively. After adsorption of $\mathrm{Mn}$ on graphene, the polarized Lowden charge values become 4.519, 0.161 and 0.765 respectively. Hence the change in magnetization is contributed by the change in polarized charge at occupation with $\Delta \mathrm{D}=$ $0.479, \Delta p=0.161$ and $\Delta s=0.760$. The minute observation of the calculations shows that the increment of magnetic moment of $\mathrm{Mn}$ graphene is due to the transference and spin rearrangement of charge at $3 \mathrm{~s} / 4 \mathrm{~s}, 3 \mathrm{p}$ and $4 \mathrm{~s}$ orbitals of $\mathrm{Mn}$ atom. We have also seen that, there is no significant rearrangement of electronic charge on carbon atom (of graphene) to contribute on changing magnetism of $\mathrm{Mn}$ graphene.

Graphene is a single atomic layer of graphite containing a honeycomb lattice of carbon atoms. Each carbon atom in the lattice, with four valence electrons, interacts with its three nearest-neighbor carbon atoms through strong bonds. The bonds are the result of the $\mathrm{sp}^{2}$ hybridization of the $2 \mathrm{~s}, 2 \mathrm{p}^{\mathrm{x}}$ and $2 \mathrm{p}^{\mathrm{y}}$ orbitals of three valance electrons. These electrons are localized, and do not contribute to electronic (transport) properties. The fourth electron occupies the $2 \mathrm{pz}$ orbital which overlaps with $2 \mathrm{pz}$ electron of neighboring Carbon atom to form $\Pi^{*}$-bond. This electron has higher energy than that of electrons which form the Sigmas bonds, and are delocalized over the entire lattice. The electronic properties of graphene are determined by these delocalized electrons. The band structure in graphene originating from pz orbital, hosting the fourth valence electron, is due to the dispersion of bonding or antibonding orbitals. The bands which correspond to the dispersion of bonding and anti-bonding molecular orbitals are called Д (valence band) and Д*(conduction band) bands.

The band structure of solid can be plotted in a usual way along the lines defined by the high symmetry points on the edges of their reducible brillouin zone. In the present work, we calculate the band structure of graphene along the path $\Gamma-\mathrm{K}-\mathrm{M}-\Gamma$ in an irreducible brillouin zone. The band structures of pure graphene is shown in Figure (5), where $x$-axis represents the symmetric points in the first brillouin zone and y-axis represents the corresponding energy values. The band structure of graphene exhibits very unique features: the conduction and the valence bands are not separated by a gap, and do not overlap either. In fact they meet at a point in the first Brillouin zone, called Dirac point. The electron dispersion in the vicinity of the Dirac points is conical, called Dirac cone. The Dirac cone and the point of intersection of two bands i.e. Dirac point, is clearly seen in the Figure 5.

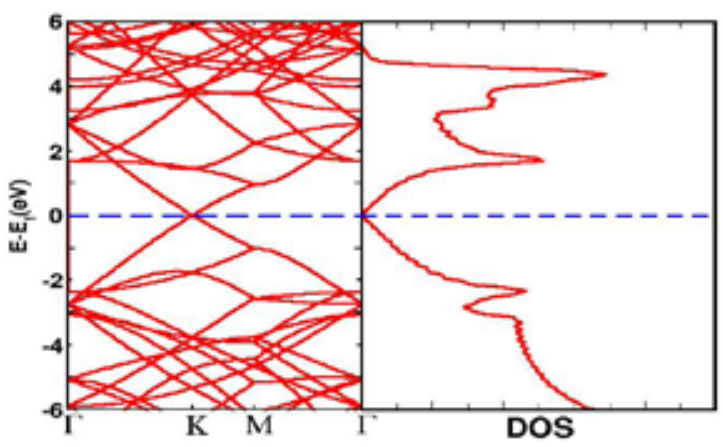

Figure 5. Band structure of the $4 \times 4$ supercell of pure graphene along $\Gamma-\mathrm{K}-\mathrm{M}-\Gamma$ path of the irreducible brillouin zone and corresponding density of states. The figure shows the zero DOS at Fermi level. 
Upon $\mathrm{Mn}$ adsorption, we find that a flat band appears just under the Fermi level (Figure 6) (the Fermi level is set to the band center and all energies are plotted relative to the Fermi level) due to majority spin bands, which corresponds to the $\mathrm{Mn} 4 \mathrm{~s}$ orbital, as can be derived from the local density of states (LDOS). In the case of minority spin, the Mn atom introduces localized states with d-orbital character near the Fermi level in the conduction band, which can also be concluded from the minority d-orbital [e.g., Geim, 2009]. The majority and minority spin channels could be noticed at PDOS plot for individual orbitals at Figure 4, however, only the majority (upspin) contribution in total DOS is shown in Figure 6. Comparing Figures 5 and 6 , it can be concluded that the conduction bands are modified and move down due to the coupling between the Mn atom and graphene

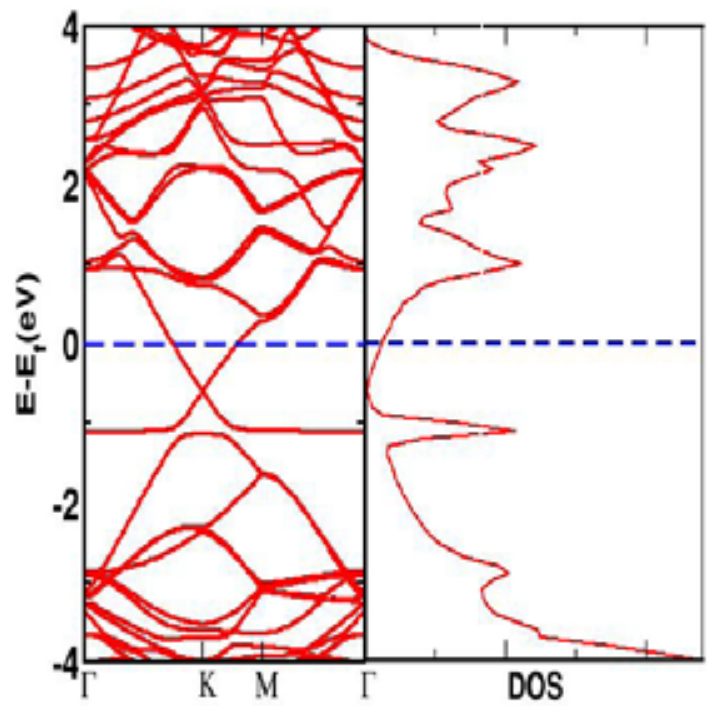

Figure 6. Band structure of the $\mathrm{Mn}$ adsorbed graphene along $\Gamma-\mathrm{K}-\mathrm{M}-\Gamma$ path of the irreducible brillouin zone and corresponding density of states. The figure shows the finite DOS at Fermi level.

\section{Conclusions and Concluding Remarks}

The functionalization of graphene by the adsorption of transition metal atom (Mn) to its surface has been investigated computationally using first-principles density-functional theory calculations within DFT-D2 level of approximations. The calculations aim to investigate the stability, electronic and magnetic properties of $\mathrm{Mn}$ decorated graphene sheet.

The values of adsorption energy of Mn at different sites of graphene suggest that (within the small difference in energy) the top site is the most favorable site for its adsorption on graphene sheet. The equilibrium distance of $\mathrm{Mn}$ atom from the surface of graphene sheet, calculated in $4 \times 4$ supercell of graphene, is found to be $2.05 \AA$ and the value agrees well with the previously reported data. The band structure analysis of the pure

Graphene and $\mathrm{Mn}$ decorated graphene systems shows that conduction and valence band meet exactly at the Fermi level in the former case. This reiterates that the pure graphene is zero band gap semiconductors. The study also observes that the conduction band and valence band overlap upon the adsorption of Mn atom. The investigation of the density of states (DOS) of pure graphene approves the non magnetic nature of pure graphene. In the case of Mn decorated graphene, however, DOS for up and down spin are not symmetrical, and indicate that the Mn decorated graphene system to be magnetic. The change of DOS of Mngraphene system is due to the rearrangement of charge distribution at $4 \mathrm{~s}, 3 \mathrm{p}$, and $3 \mathrm{~d}$ orbitals of Manganese atom. The study reveals that the London dispersion interaction contributes 
the major binding strength in such type of adsorption. Further investigation can be done to check the adsorption properties of $\mathrm{Mn}$ atom/s nearby more reactive parts of graphene plane like towards zig-zag graphene edges. Other form of van der Waal's interaction, in addition to London dispersion corrections, can be searched in this type of weak bindings.

\section{Acknowledgments}

The authors acknowledge the useful discussion with Prakash Parajuli. We also acknowledge the partial support from the Abdul Salam International Centre for Theoretical Physics (ICTP) through office of external activities within NET-56. Further we appreciate TWAS Research Grants for providing support for computational facilities. Editor N. P. Chapagain thanks the reviewers for their assistance in evaluating this paper.

\section{References}

AlZahrani, A. Z. (2012), Theoretical investigation of manganese adsorption on graphene: A firstprinciples comparative study, Physica B. 407, 992-1002.

Belbase, K. (2013), First-principal study of adsorption of hydrogen molecule on sodium adsorbed graphene, for the partial fullment of the requirement for master's degree of science in physics, CDP, TU, Kirtipur, Nepal.

Castro Neto, A. H. (2010), The carbon new age, Department of Physics, Materials today 13 (3), 1-4. (http://arxiv.org/ftp/arxiv/ papers/1003/1003.4520.pdf)

Castro Neto, A. H ., F. Guinea, and N. M. R. (2007), Peres, Phys. World 19, 33,.

Chan, K. T., J. B. Neaton, and M. L. Cohen (2008), First-principles modeling of the interactions of iron impurities with graphene and graphite, Phys. Rev. B 77, 235430.

Compton, O., S. Nguyen (2010) Graphene Oxide,
Highly Reduced Graphene Oxide, and Graphene: Versatile Building Blocks for Carbon Based Materials 6, 711-723.

Ding, J., Z. Qiao, W. Feng, Y. Yao and Q. Niu (2011), Engineering quantum anomalous/valley Hall states in graphene via metal-atomadsorption: Anab-initio study, Phys. Rev. B 84, 195444 doi:10.1103/Phys Rev B. 84.195444.

Fair, K. M., X. Y. Cui, L. Li, C. C. Shieh, R. K. Zheng, Z. W. Liu, B. Delley, M. J. Ford, S. P. Ringer (2013), Hydrogen adsorption capacity of adatoms on double carbon vacancies of graphene: A trend study from first principles, Phys. Rev. B 87, 014102 doi.org/10.1103/ PhysRevB.87.014102.

Ferrari, L., J. Kaufmann, F. Winnefeld, J. Plank (2010), Interaction of cement model systems with superplasticizers investigated by atomic force microscopy, zeta potential, and adsorption measurements, Journal of Colloid and Interface Science 347, 15.

Fletcher, R. (1987), Practical Methods of Optimization, Wiley, New York.

Geim, A. K. (2009) Graphene: Status and Prospects, Science 324, 1530-1534 DOI: 10.1126/ science. 1158877 .

Giannozzi, P., et. al. (2009) QUANTUM ESPRESSO: a modular and open-source software project for quantum simulations of materials, J. Phys. Condence. Matter 21, 395502 doi:10.1088/0953-8984/21/39/395502.

Hohenberg, P. and W. Kohn (1964), Inhomogeneous Electron Gas Phys. Rev. B 136, 864, doi. org/10.1103/PhysRev.136.B864.

Khaniya, A. (2013), First-principal study of adsorption of hydrogen molecule on palladium adsorbed graphene, for the partial fulment of the requirement for master's degree of science in physics, CDP, TU, Kirtipur, Nepal.

Kohn, W., and L. J. Sham (1965), Self-Consistent Equations Including Exchange and Correlation Effects. Phys. Rev. 140, 1133.doi.10.1103/ PhysRev.140.A1133. 
Lamichhane, S. et. al. (2014), Hydrogen storage on Platinum decorated graphene: A first-principles study, Bibechhana 11 , 107-117.

Lee, C., X. Wei, J. W. Kysar, J. Dordrecht (2008), A. M. Mannion, Carbon and Its Domestication Springer, The Nether-lands, Hone, Science 321, 385.

Mao, Y., J. Yuan, J. Zhong (2008) Density functional calculation of transition metal adatom adsorption on graphene, J. Phys.: Condens. Matter 20 , 115209, doi:10.1088/0953-8984/20/11/115209.

Mao, Y., J. Zhong (2008), Structural, electronic and magnetic properties of manganese doping in the upper layer of bilayer graphene Nanotechnology, IOP 19, 205708, doi:10.1088/09574484/19/20/205708.

Marzari, N., D. Vanderbilt, A. de Vita, and M. C. Payne (1999), Deliberately Designed Materials for Optoelectronics Applications, Phys. Rev. lett. 82 doi.org/10.1103/PhysRevLett.82.3296.

Medeiros, P. V. C., F de Brito Mota, Artur J. S. Mascarenhas and C. M. C. de Castilho (2010), A DFT study of halogen atoms adsorbed on graphene layers, IOP Sci. 21, 115701 doi:10.1088/0957-4484/21/48/485701.

Monkhorst, J. H., D. J. Pack (1940), Computational Methods for Large Systems: Electronic Structure, Phys. Rev. B 16, 1748

Moritomo Y. et. al. (1996), Cobalt Oxides: From Crystal Chemistry to Physics, Chem. Soc. Rev. 380, 141.

Murali, R., Y. Yang, K. Brenner, T. Beck, and J.D. Meindl, Appl. (2009) Breakdown current density of graphene nanoribbons Phys. Lett. 94, 243114.

Novoselov, K. S., A. K. Geim, S. V. Morozov, D. Jiang, M. I. Katsnelson, Y. Zhang, I. V. Grigorieva, S. V. Dubonos and A. A. Firsov (2005). Two-dimensional atomic crystals, Nature 438, 197.
Novoselov, K. S., A. K. Geim, S. V. Morozov, D. Jiang, M. I. Katsnelson, S. V. Dubonos, I. V. Grigorieva and A. A. Firsov (2004), Electric Field Effect in Atomically Thin Carbon Films, Science 306, 666-669.

Novoselov, K. S., Z. Jiang, Y. Zhang, S. V. Morozov, H.L.stormer, U. Zeitler, J.C. Maan, G. S. Boebinger, P. Kim, and A. K. Geim (2007), Room-Temperature Quantum Hall Effect in Graphene, Science 315, 1379.

Pantha, N., K. Belbase, N. P. Adhikari (2014), Firstprinciples study of the interaction of hydrogen molecular on Na-adsorbed graphene, Appl. Nanosci. doi 10, 1007.

Perdew, J. P., K. Burke, and M. Ernzerhof (1996), Generalized Gradient Approximation Made Simple Phys. Rev. Lett. 77, 3865 doi.org/10.1103

Sholl, D. S., J. A. Steckel (2009), Density functional theory, A practical introduction, John Willy \& Sons Publication, USA.

Thijssen, M. (1999), Computational Physics, Cambridge University Press, Cambridge.

Valencia, H., A. Gil, and G. Frapper (2010), Trends in the Adsorption of 3d Transition Metal Atoms onto Graphene and Nanotube Surfaces: A DFT Study and Molecular Orbital Analysis, J. Phys. Chem. C 114, 14141-14153, doi: 10.1021/ jp103445v.

Wolf, E. L., (2013) Application of graphene , Springer, Brooklyn, USA.

Wu, M., C. Cao and J. Z. Jiang (2010), Electronic structure of substitutionally Mn-doped graphene, Phys. 12, 063020, doi:10.1088/13672630/12/6/063020.

Zhang, T Y., T. Tang, C. Girit, Z. Hao, M. C. Martin, A. Zettl, M. F. Crommie, Y. R. Shen and F. Wang (2009), Direct observation of a widely tunable bandgap in bilayer graphene, Nature 459, 820823. 